\title{
Dermosccopic features of progressive cribriform and zosteriform hyperpigmentation
}

\section{Subrata Malakar', Samipa Samir Mukherjee ${ }^{2}$}

\author{
${ }^{1}$ Department of Dermatology, Rita Skin Foundation, Kolkata, India, ${ }^{2}$ Department of Dermatology, Cutis Academy of \\ Cutaneous Sciences, Bangalore, India
}

Corresponding author: Dr. Samipa Samir Mukherjee, E-mail: drsamipamukherjee@gmail.com

Sir,

Progressive cribriform and zosteriform hyperpigmentation (PCZH), first described by Rower et al. in 1978, is a distinctive clinicopathological pigmentary disorder of the skin [1]. PCZH refers to an asymptomatic dermatosis consisting of a single area of uniformly tan, cribriform, macular pigmentation in a zosteriform distribution; histological pattern consisting of mild increase in melanin pigment in basal cell layer and complete absence of nevus cells. It had onset well after birth with no reported systemic associations. Di Lernia et al. [2], suggested that PCHZ should be considered as a part of spectrum of Linear and Whorled Nevoid Hyperpigmentation(LWNH) as apart from later age of onset, there was no difference between the two disorders clinically and histopathologically. They also proposed the terms 'Diffuse LWNH' for previous reported cases of LWNH and 'Localized-form' for PCZH [3].

An otherwise healthy 30 year old lady presented to the dermatology outpatient department of with a fouryear history of asymptomatic dark patches confined to the right side of her arm. Brownish macules with a cribriform configuration extended linearly from the lower part of right upper arm to the fore arm area on the dorsal and ventral side (Fig. 1). Examination of her hair, nails, and mucosae did not reveal any abnormality. The lesions had first appeared over the upper arm, four years earlier, and had gradually progressed in linear fashion to their present extent. There was no history of trauma, inflammation, or use of any topical application on the involved areas prior to development of the lesions. Past medical history and family history were non-contributory. Our clinical differential diagnoses included lichen planus pigmentosus, verrucous epidermal nevus, reticulate pigmentary disorder,non hypertrichotic variant of Becker's nevus and linear and whorled nevoid hypermelanosis.

Overview of the dermoscopic image reveals small islands of accentuated pigment (Fig. 2). Dermoscopy

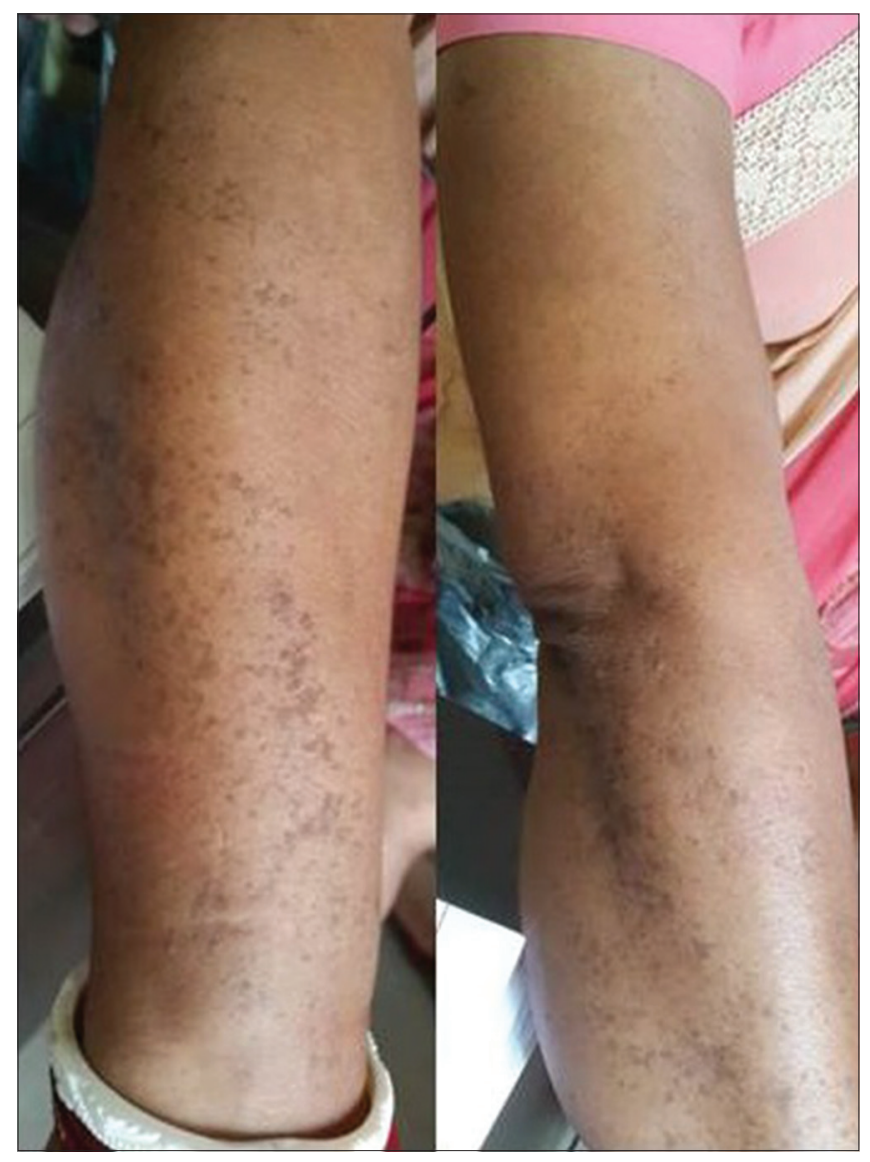

Figure 1:Brownish macules with a cribriform configuration extended linearly from the lower part of right upper arm to the fore arm area on the dorsal and ventral side

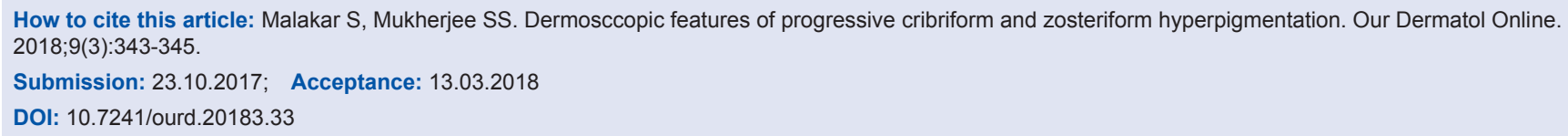




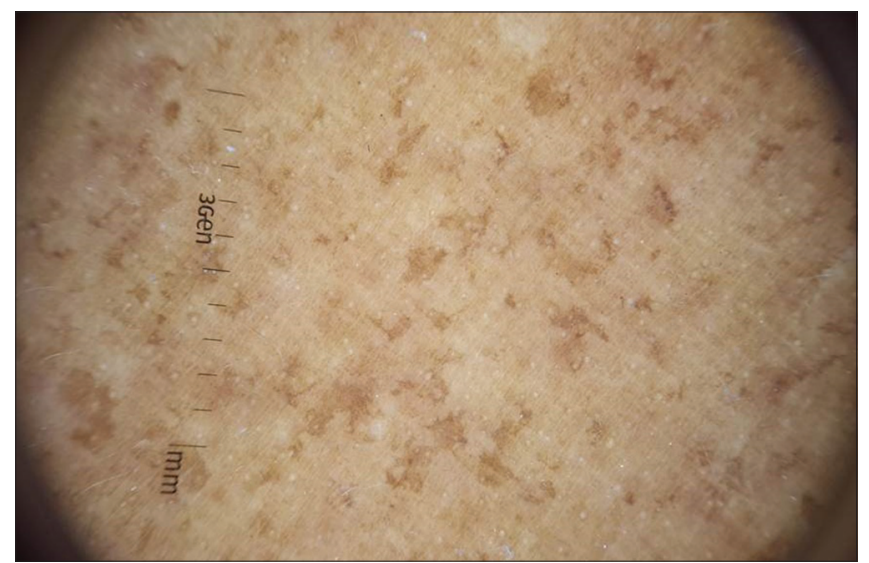

Figure 2: Dermoscopic overview showing small islands of accentuated pigment

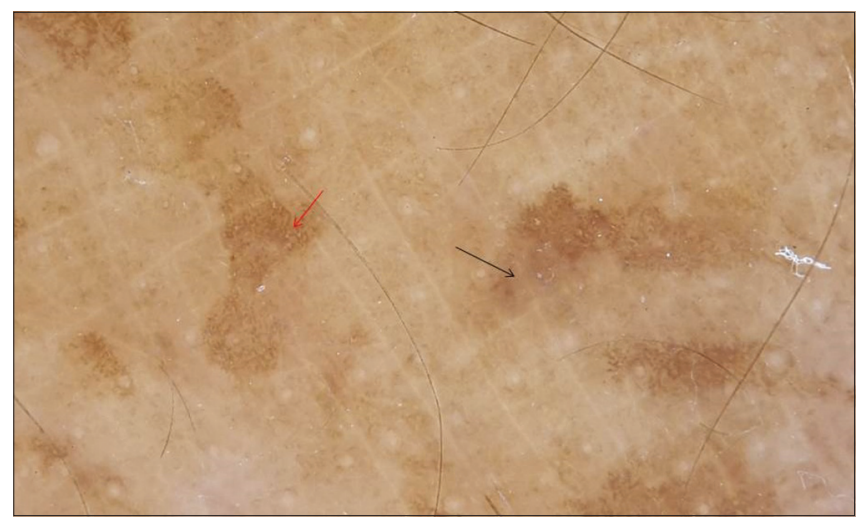

Figure 3: Dermoscopy of the hyperpigmented areas revealed accentuation of melanin network, areas with greyish homogenous hyperpigmentation and brown dots and globules

of the hyperpigmented areas revealed accentuation of melanin network, areas with greyish homogenous hyperpigmentation and brown dots and globules (Figs. 3 and 4a). Hyperpigmented lesions may show finger print like structures (Fig. 4b). Rosettes were noted occasionally.

A skin biopsy from a representative forearm lesion revealed elongated epidermal rete ridges with hypermelanisation of the basal layer. Pigmentary incontinence was noted in focal areas. Melanophages, and dermal inflammatory infiltrate were not seen. Also, nevus cells were completely absent. Lichen planus was ruled out based on the absence of basal layer degeneration and interface dermatitis. Epidermal nevus could be reliably excluded due to the lack of characteristic epidermal changes.

Based on clinicodermoscopicpathological correlation, we made a diagnosis of progressive cribriform and zosteriform hyperpigmentation.

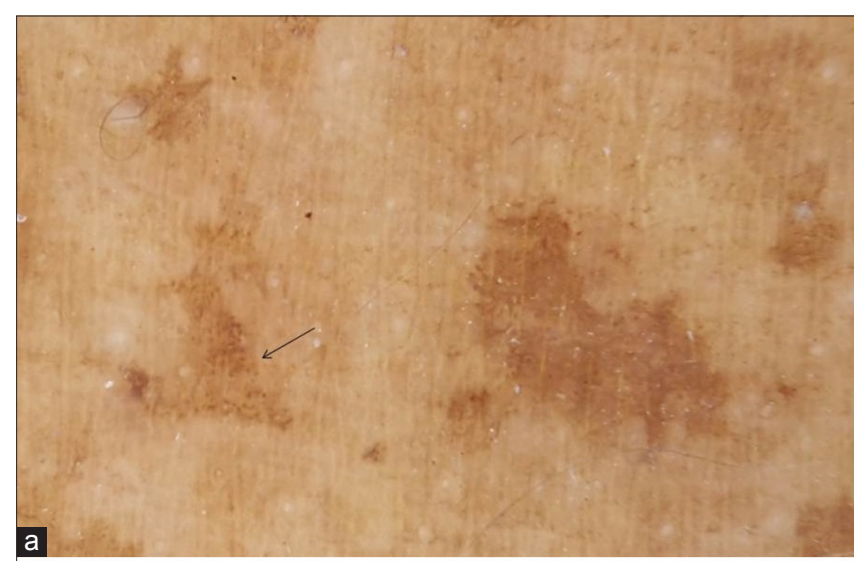

b

Figure 4: (a) Dermoscopy of the hyperpigmented areas revealed accentuation of melanin network, areas with greyish homogenous hyperpigmentation and brown dots and globules, (b) Hyperpigmented lesions showing finger print like structures

This distinctive pigmentary dermatosis has five diagnostic criteria: (a) uniformly tan cribriform macular pigmentation in a zosteriform distribution, (b) histology showing an increase in basal layer melanin along with complete absence of nevus cells, (c) absence of history of rash, injury, or inflammation to suggest post-inflammatory hyperpigmentation, (d) onset well after birth with gradual extension, and (e) lack of other associated cutaneous or internal abnormalities $[1,4]$.

The etiopathogenesis of progressive cribriform and zosteriform hyperpigmentation is possibly related to somatic mosaicism that develops during embryogenesis [5]. Another hypothesis postulated stated that clonal migration and proliferation of embryonic melanoblasts along the Lines of Blaschko could be the underlying mechanism behind this condition.

Histopathological correlate of hypermelanisation of rete ridges points towards the dermoscopic accentuation of the melanin network. Areas of greyish homogenous pigmented areas correlates with areas of 
pigment incontinence. Pigment incontinence has been reported in 13/30 cases by Cho et al [6]. The occurrence of rosettes could be attributed to the actinic area of distribution of lesions. This can be differentiated from lichen planus pigmentosus where the melanin network remains uncharacterized and pigmentary pattern is characterized by the presence of brown dots and globules with either diffuse or mixed pattern of pigmentation.

The dermoscopic description of this entity has not been described in literature so far and doesnot find its position even in the diagnostic criteria. We propose that a constellation of accentuation of melanin network, areas with greyish homogenous hyperpigmentation, finger print like structures along with clustered brown dots and globules could provide a clue towards this entity.

To the best of our knowledge this is the first ever time that the dermoscopic description of this entity is being reported and we request continuous observations of these findings in different skin type and further documentation of the same to establish it as one of the diagnostic criteria for this uncommon entity.

\section{CONSENT}

The examination of the patient was conducted according to the Declaration of Helsinki principles.

\section{REFERENCES}

1. Rower JM, Carr RD, Lowney ED. Progressive cribriform and zosteriform hyperpigmentation. Arch Dermatol. 1978;114:98-9.

2. Di Lernia V, Patrizi A, Neri I, Varotti C. Reticulate hyperpigmentation of Iijima, Naito and Uyeno and other linear hyperpigmentations of children. Acta Derm Venereol. 1992;72:393.

3. Di Lernia V. Linear and Whorled Hypermelanosis. Pediatr Dermatol. 2007;24:205-10.

4. Hassan I, Rather PA, Sajad P. Linear lichen planus pigmentosus and coincidental ipsilateral facial nerve palsy: An unusual observation. Our Dermatol Online. 2012;3:361-2.

5. Schacht JP, Farnworth E, Hogue J, Rohena L. Tetraploid-diploid mosaicism in a patient with pigmentary anomalies of hair and skin: a new dermatologic feature. Clin Case Rep. 2017;6:103-8.

6. Cho E, Cho SH, Lee JD. Progressive cribriform and zosteriform hyperpigmentation: A clinicopathologic study. Int J Dermatol 2012;51:399-405.

Copyright by Subrata Malakar, et al. This is an open-access article distributed under the terms of the Creative Commons Attribution License, which permits unrestricted use, distribution, and reproduction in any medium, provided the original author and source are credited. Source of Support: Nil, Conflict of Interest: None declared. 